\title{
Experimental Infection of Sheep With Pseudorabies Viruses Isolated In Shanghai, China, Between 2010 and 2012
}

\author{
Houbin Ju${ }^{\dagger}$, Dequan Yang ${ }^{\dagger}$, Jian Wang, Peihong Liu, Xinyong Qi, Jian Liu, Xianchao Yang, Bo Deng, \\ Feifei Ge, Xiujuan Wu, Xin Li, Kaihang Li and Jinping Zhou*
}

Shanghai Animal Disease Control Center, Shanghai 201103, China

${ }^{\dagger}$ Equal contributors

*Corresponding author: Jinping Zhou, Shanghai Animal Disease Control Center, 855 Hongjing Road, Shanghai 201103, China, Tel: +86 2162696318 ; Fax: +86 21 62696318; E-mail: shzjpvet@163.com

Received date: September 18, 2017; Accepted date: October 06, 2017; Published date: October 13, 2017

Copyright: (c) $2017 \mathrm{Ju} \mathrm{H}$, et al. This is an open-access article distributed under the terms of the Creative Commons Attribution License, which permits unrestricted use, distribution, and reproduction in any medium, provided the original author and source are credited.

Citation: Ju H, Yang D, Wang J, Liu P, Qi X, et al. (2017) Experimental Infection of Sheep With Pseudorabies Viruses Isolated In Shanghai, China, Between 2010 and 2012. Eur Exp Biol. Vol. 7 No. 5:30.

\section{Abstract}

For many years, pseudorabies has been efficiently controlled in China by vaccination. However, a sudden outbreak occurred on many pig farms during 2012-2013. In this study, phylogenetic analysis was carried out with the aim of understanding the genetic relatedness between strains in both populations and comparing strains in China with those circulating in other countries. To obtain more pathogenetic relevant data regarding two pseudorabies virus (PRV) isolates (SH2010 and SH2012) in sheep, we evaluated samples of PRV isolated in Shanghai, China between 2010 and 2012. Based on clinical signs, body temperature variation, and the maximum amounts of viral DNA in unvaccinated/infected sheep, the emerging PRV variant strain, SH2012 was observed to be more highly virulent than the traditional PRV SH2010 strain. The data of the present study will aid in a better understanding of the differences in pathogenicity between the traditional PRV SH2010 strain and the emerging PRV variant strain SH2012.

Keywords: Pathogenicity; Pseudorabies virus; Sheep; Variant strain

\section{Introduction}

Pseudorabies virus (PRV), which belongs to the family Herpesviridae, subfamily Alphaherpesvirinae, and genus Varicellovirus, is the causative agent of Aujeszky's disease [1]. PR is an economically significant disease in domestic swine in China. PRV was first observed in China in 1947 and was found to be epidemic in pigs in the 1960s. In response, the Bartha-K61 vaccine strain was introduced to China in the late 1970s [2]. From the 1990s to 2010, the Bartha-K61 vaccine was widely used in Chinese pig herds and PR was effectively controlled [3,4]. However, in October 2011, a severe PRV outbreak occurred in Bartha-K61-vaccinated pig herds on many Chinese farms, resulting in considerable economic losses [5-8]. The causative agent of the outbreaks was confirmed to be a PRV variant $[3,9]$. The PRV variant was shown to be both antigenically different from the classical PRVs and more pathogenic in older pigs $[3,7,8,10]$. Previous studies on immunological protection in sheep have shown that the Bartha-K61 vaccine does not provide complete protection against the emerging PRV variant, but could offer full protection against infection with PRV strain SC (an earlier virulent strain in China) $[3,11]$.

In this study, we genetically characterized two PRV strains (SH2010 and SH2012) from domestic swine using phylogenetic analysis to gain clearer insight into the genetic relatedness between strains in both populations and to compare Chinese strains with those circulating in other countries. To obtain more pathogenetic relevant data regarding the two PRV isolates (SH2010 and SH2012) in sheep, we evaluated PRV samples isolated in Shanghai, China between 2010 and 2012

\section{Material and Methods}

\section{Viruses and cells}

PRV strain SH2010 was isolated from unvaccinated pigs on an infected farm in Shanghai, China, in 2010. PRV strain SH2012 was isolated in May 2012 from neonatal piglets showing severe neurological signs, including tremors, convulsions, and opisthotonus, during a PR outbreak on a pig farm that rou-tinely used the commercial Bartha-K61 vaccine. All viruses were propagated in baby hamster kidney 21 (BHK-21) cells in Dulbecco's modified Eagle's medium supplemented with $10 \%$ fetal bovine serum (Gibco BRL, Grand Island, NY, USA) at $37^{\circ} \mathrm{C}$ with $5 \% \mathrm{CO}_{2}$ in a humidified incubator. Cell cultures were passaged at least four times to obtain a sufficient yield of the virus for subsequent characterization.

\section{Sequencing and phylogenetic analysis of the $\mathrm{gC}$ gene}

For sequencing purposes, part of the gC gene of the viral isolates was amplified with PrimeSTAR HS DNA Polymerase and GC Buffer (Takara, Dalian, China) using previously published 
primers [12]. An amplicon of the expected size was excised from an agarose gel, and the DNA was purified using the QIA quick Gel Extraction Kit (Qiagen, Hilden, Germany). The PCR products were cloned into the pEASY-Blunt Zero plasmid and sequenced by Invitrogen (Shanghai, China). The sequences obtained were submitted to GenBank (accession numbers KY569296 and KY569297). Sequences were aligned using the Muscle algorithm in MEGA 5.0 software [13]. Phylogenetic trees were constructed using the neighbor-joining algorithm, with 1,000 bootstrap repetitions, and the Kimura 2-parameter substitution model.

\section{Vaccine}

The PRV Bartha-K61 vaccine with a virus titer of $10^{5.0}$ $\mathrm{TCID}_{50}$ /dose was purchased from Harbin Pharmaceutical Group Bio-vaccine Co., Ltd. (Harbin, China). According to the quality standards for this vaccine [14], the virus titer of the qualified product is $>5,000 \mathrm{TCID}_{50} /$ dose.

\section{Animal experiments}

Sheep are commonly used to examine the efficacy of the Bartha-K61 vaccine in China. The experimental design, immune dosage, and viral challenge were in accordance with China's quality standards for veterinary biological products [14]. Sixteen 6-month-old sheep were obtained from a farm that was determined to be free of PRV infection, based on real-time PCR (Anheal, Beijing, China) and glycoprotein E and gB ELISAs (IDEXX, Westbrook, ME, USA).

The sheep were divided into three groups: group $A$ (seven sheep), group B (seven sheep), and group C (two sheep) (Table 1). Four sheep (vaccinated/infected) in groups $A$ and $B$ were injected intra-muscularly with $10^{5.0} \mathrm{TCID}_{50}$ of the Bartha-K61 vaccine, whereas the other three sheep (unvaccinat-ed/infected) in groups A and B were not vaccinated. Sheep in group C (unvaccinated/uninfected) were injected with $1 \mathrm{~mL}$ of phosphate-buffered saline (PBS, pH 7.4). After continuous observation for 14 days, sheep in group $A$ were inoculated intramuscularly with $1,00050 \%$ lethal doses $\left(L_{50}\right)$ of the PRV SH2010 strain (vaccinated/infected SH2010), and sheep in group B were challenged with $1,000 L_{50}$ of the SH2012 strain (vaccinated/infected SH2012). The sheep in group C were injected intramuscu-larly with $1 \mathrm{~mL}$ of the BHK-21 cell culture supernatant as a mock group (vaccinated/uninfected). The $\mathrm{LD}_{50}$ was titrated according to established quality standards [14].

Based on the quality standards of the Bartha-K61 vaccine, at least two sheep in the unvaccinated group should have become ill with PR, leading to death, and all vaccinated sheep should have been protected against infection [14].

Table 1: experimental design (Vaccination and challenge data).

\begin{tabular}{|c|c|c|c|c|c|c|}
\hline \multicolumn{2}{|l|}{ Groups } & $\begin{array}{l}\text { Sheep } \\
\text { amounts }\end{array}$ & Vaccine & $\begin{array}{l}\text { Vaccine doses } \\
\left(T_{C I} D_{50}\right)\end{array}$ & Challenge virus & $\begin{array}{l}\text { Challenge } \\
\text { doses }\left(L_{50}\right)\end{array}$ \\
\hline \multirow{2}{*}{ Group A } & vaccinated/infected & 4 & Bartha K61 & $10^{5.0}$ & SH2010 & $10^{3.0}$ \\
\hline & unvaccinated/infected & 3 & & & SH2010 & $10^{3.0}$ \\
\hline \multirow{2}{*}{ Group B } & vaccinated/infected & 4 & Bartha K61 & $10^{5.0}$ & SH2012 & $10^{3.0}$ \\
\hline & unvaccinated/infected & 3 & & & SH2012 & $10^{3.0}$ \\
\hline Group C & unvaccinated/uninfected & 2 & PBS & $1 \mathrm{ml}$ & BHK-21 supernatant & $1 \mathrm{ml}$ \\
\hline
\end{tabular}

Clinical signs were checked daily throughout the study, and rectal temperatures were recorded daily before feeding. Blood samples were taken from 1 to $14 \mathrm{DPI}$, and nasal swabs were taken daily (in $1.5 \mathrm{ml}$ of PBS, 1-14 DPI) for detection of PRV DNA. Tissue samples were resected from the brains and internal organs of sheep that died for viral detection using real-time PCR, virus isolation, and histo-pathological examination. The experiment was conducted under biosafety level 2 conditions, and it was reviewed and approved by the Committee for Ethics and Animal Welfare.

\section{Results}

\section{Phylogenetic analyses based on gC}

The gC genes of PRV were amplified and sequenced from the two isolates. As shown in Figure $\mathbf{1 A}$, the two isolates both shared an insertion of seven amino acids (AAASTPA) between positions 63 and 69 in the $\mathrm{gC}$ gene, as compared to the classic PRV strains Bartha, Becker and Kaplan; only earlier Chi-nese strains, emerging variant strains, and the P-Prv strain shared these insertions. Based on a phylo-genetic analysis of the $\mathrm{gC}$ gene, the two major genotypes observed were designated as genotype I and genotype II (Figure 1B); these results were similar to those reported in the literature [15]. Genotype I was composed of viruses from a wide range of geographic regions, including Europe, America, and Asia. Genotype I could be subdivided into six subgroups as follows: subgroup 1.1 consisted mostly of strains isolated in Germany, France, Belgium, and Spain; subgroup 1.2 comprised strains isolated in France; subgroup 1.3 showed a high degree of diversity, consisting of American (United States and Argentina) and European (Italy and Sweden) strains; subgroup 1.4 contained strains isolated in Europe and South America; strains of subgroup 1.5 were all isolated in Hungary, including the Shope and Kaplan isolates; and sub-group 1.6 comprised European and Asian strains, with the Chinese strains most likely being vaccine-derived, as they are closely related to the Bartha strain (a widely used vaccine strain). Geno-type II consisted mainly of Chinese strains that were generally closely related and clustered together. Most of the emergent strains isolated after 2011, including SH2012, were closely and independently clustered together, among which 
several emergent strains as well as four earlier strains (Ea, LXB6, LXB88, and HUYD) comprised a small subgroup. Unlike the close relatedness of the emergent strains, the earlier strain $\mathrm{Fa}$ (isolated in 1964) and SH2010 were phylogenetically diverged from the four earlier strains referred to above and differed distinctly from the emergent strains (Figure 1B).

\begin{tabular}{|c|c|c|c|c|c|c|c|c|c|c|}
\hline \multirow{2}{*}{ Majority } & \multicolumn{6}{|c|}{ MASLARAMLALLLALYTAAIAAAASSSTTALGTT PNGGGGGNSSAGELSPSPPSTPEPVSGTTG } & $\overline{\text { A.ASTPA }}$ & \multicolumn{3}{|c|}{ AVSTPRVPPPSVSRRKPQRNGNRTRVHGDKA } \\
\hline & 10 & 20 & 30 & 40 & 50 & 60 & & 80 & 90 & 100 \\
\hline SH2010 & $\ldots \ldots \ldots \ldots$ & $\ldots$ & $\ldots$ & ... & ... & AP. & & $\ldots \ldots$ P...... & $\cdots$ & \\
\hline $\mathrm{SH} 2012$ & $\ldots \ldots \ldots$ & $\cdots$ & $\ldots$ & $\cdots$ & & $\cdots$ & & (9. & & $\cdots$ \\
\hline HeN1 2012 China & $\ldots \ldots \ldots$ & ... & $\ldots$ & ... & ... & . & & .... & $\cdots$ & \\
\hline JS-2012 China & & - & 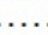 & $x_{-1}+2+2$ & $\cdots$ & & & & * & \\
\hline TJ 2012 China & $\ldots \ldots \ldots$ & $\cdots$ & $\cdots$ & . & . & & & & & \\
\hline Ea 1999 China & n.m. & ... & $\cdots$ & ... & .. & & & ......... & . & E. \\
\hline LXB88 2005 China & $\ldots \ldots \cdots \cdots$ & $\ldots$ & $\cdots$ & ... & $\ldots s$ & & & & & E. \\
\hline HUYD-China-2008 & $\ldots \ldots \ldots$ & ... & ... & ... & $\cdots$ & & & & & .E. \\
\hline Fa 1964 China & $\ldots \ldots \ldots$ & $\ldots$ & $7 \ldots$ & . & & & & $\ldots$.... & & .R. \\
\hline P-PrV 2003 Malaysia & $\ldots \ldots \ldots \ldots$ & $\ldots$ & $\ldots$ & $\ldots$ & $\ldots$ & & & $\ldots \ldots$.... & 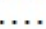 & $\ldots$ \\
\hline Kaplan 1959 Hungary & $\ldots \ldots \ldots \ldots$ & $\cdots$ & $\cdots$ & $\ldots$ & ... & PEA. & & ........ & ..N. & ... \\
\hline Bartha 1961 Hungary & $\ldots \ldots \ldots \ldots$ & $\cdots$ & $\cdots$ & $\ldots$ & $\cdots$ & PEA. & & ........ & ..N. & \\
\hline Becker 1970 USA & n.m. & & D. & & & PEA. & & ,..P..... & ..N. & \\
\hline
\end{tabular}

B

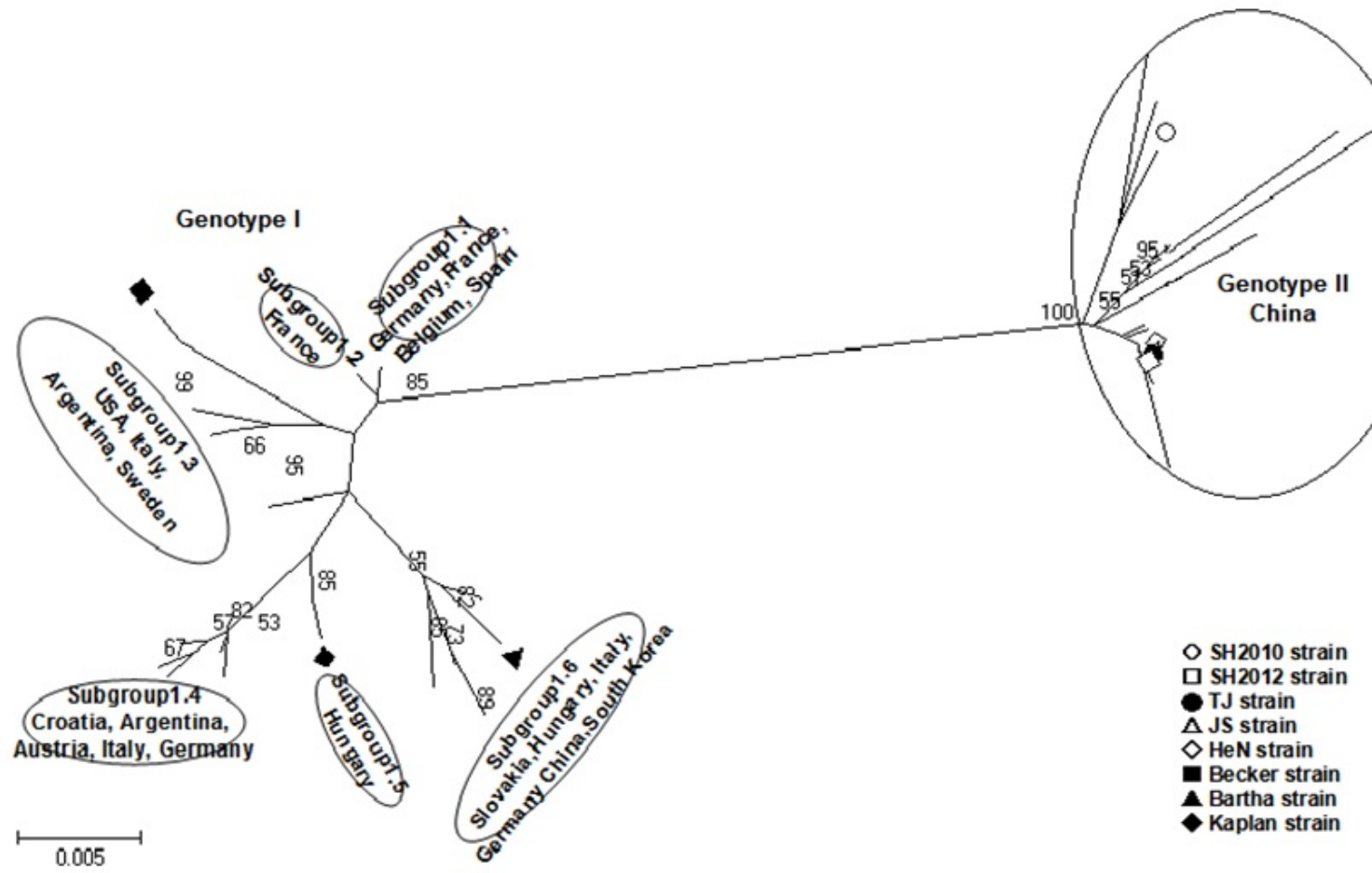

Figure 1: (A) Alignment the partial sequences of glycoprote in gC gene of PRV at the nucleotide level. Black box indicates the region of insertion. (B) Phylogenetic analysis of the PRV genome based on gC sequences. A phylogenetic tree was constructed at the nucleotide level, based on the partial gC gene sequences of strains isolated from all over the world. The ellipse represents the number of strains represented. The countries from which the PRV strains were isolated are marked beside the ellipse. 0 Represents SH2010, represents SH2012, represents TJ, $\Delta$ represents JS, $\diamond$ represents HeN1, represents Becker, represents Bartha and represents Kaplan.

\section{Clinical signs}

Following inoculation, the clinical condition of all animals was observed daily. As shown in Table 2, vaccinated sheep challenged with the $\mathrm{SH} 2010$ strain in group A showed no signs of disease and were all protected against infection. The three unvaccinated/infected sheep in group $A$ showed signs of depression, incoordination, and severe neurological deficits, such as "mad itch," as well as fever $\left(40.5^{\circ} \mathrm{C}\right)$ on day $5 \mathrm{PI}$. These animals died on days 6 and $7 \mathrm{PI}$. However, two of the four vaccinated sheep chal-lenged with the $\mathrm{SH} 2012$ strain in group $\mathrm{B}$ displayed clinical signs of PR, and one died. Clinical signs were first noticed in the three unvaccinated/infected sheep in group $B$ as early as 2 days post-infection (DPI). All the sheep had a high fever $\left(41.0^{\circ} \mathrm{C}\right)$, poor appetite, showed clinical signs of $P R$, and died within 4-6 days. Sheep of group $C$ showed no clinical signs 
throughout the experiment, neither was any rise in rectal temperature observed (Figure 2).

Table 2: Protective efficacy of the Bartha-K61 vaccine in sheep when challenged with the PRV SH2010 or SH2012 strain.

\begin{tabular}{|c|c|c|c|c|c|c|}
\hline $\begin{array}{l}\text { Group } \\
\text { No. }\end{array}$ & Vaccine & $\begin{array}{l}\text { No. PRV antibody- } \\
\text { positive before } \\
\text { challenge/no. total }\end{array}$ & Challenge virus & $\begin{array}{l}\text { No. sick after challenge/ } \\
\text { No.total }\end{array}$ & $\begin{array}{l}\text { No. sick after } \\
\text { challenge/no. Total }\end{array}$ & $\begin{array}{l}\text { No. protected } \\
\text { sheep/No. total }\end{array}$ \\
\hline \multirow[t]{2}{*}{ Group A } & Bartha K61 & $4 / 4$ & SH2010 & $0 / 4$ & $0 / 4$ & $4 / 4$ \\
\hline & None & $0 / 3$ & $\mathrm{SH} 2010$ & $3 / 3$ & $3 / 3$ & $0 / 3$ \\
\hline \multirow[t]{2}{*}{ Group B } & Bartha K61 & $4 / 4$ & SH2012 & $2 / 4$ & $1 / 4$ & $3 / 4$ \\
\hline & None & $0 / 3$ & SH2012 & $3 / 3$ & $3 / 3$ & $0 / 3$ \\
\hline Group C & PBS & $0 / 2$ & $\begin{array}{l}\text { BHK-21 } \\
\text { supernatant }\end{array}$ & $0 / 2$ & $0 / 2$ & $2 / 2$ \\
\hline
\end{tabular}

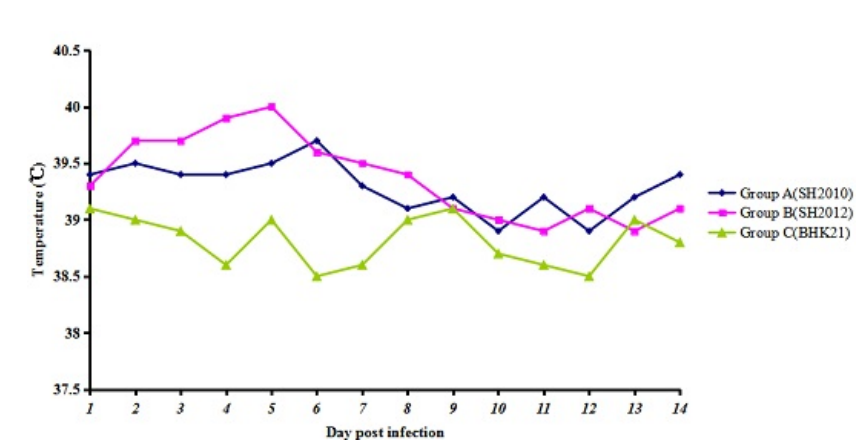

Figure 2: Rectal temperatures of sheep inoculated with two different strains of PRV. Sixteen 6-month-old sheep were inoculated with three doses, $1000 \mathrm{LD}_{50} \mathrm{SH} 2010$ (group A), 1000 LD $_{50}$ SH2012 (group B) and BHK21 cell culture supernatant (group C). The rectal temperatures of these sheep were monitored daily. Data were presented as mean \pm SD.

\section{Serology}

Antibodies were detected using commercial enzyme-linked immunosorbent assay (ELISA) kits. Following inoculation, PRV glycoprotein $B(\mathrm{gB})$ antibodies were detected in the serum samples of all sheep (Figures 3A-3C). PRV gB antibodies were not evident in any of the sheep before challenge, but were subsequently produced in all immunized sheep over 2 weeks post-vaccination and increased pro-gressively after challenge (Figure 3A). Levels of PRV gB antibodies in vaccinated/infected SH2012 sheep in group B were higher than those of PRV gB antibodies in vaccinated/infected $\mathrm{SH} 2010$ sheep in group $A$ (Figure 3B). PRV gB antibodies were not detected in any unvaccinated/infected sheep in groups A or B that were inoculated with either SH2010 or SH2012 during the experiment (Figure 3C).

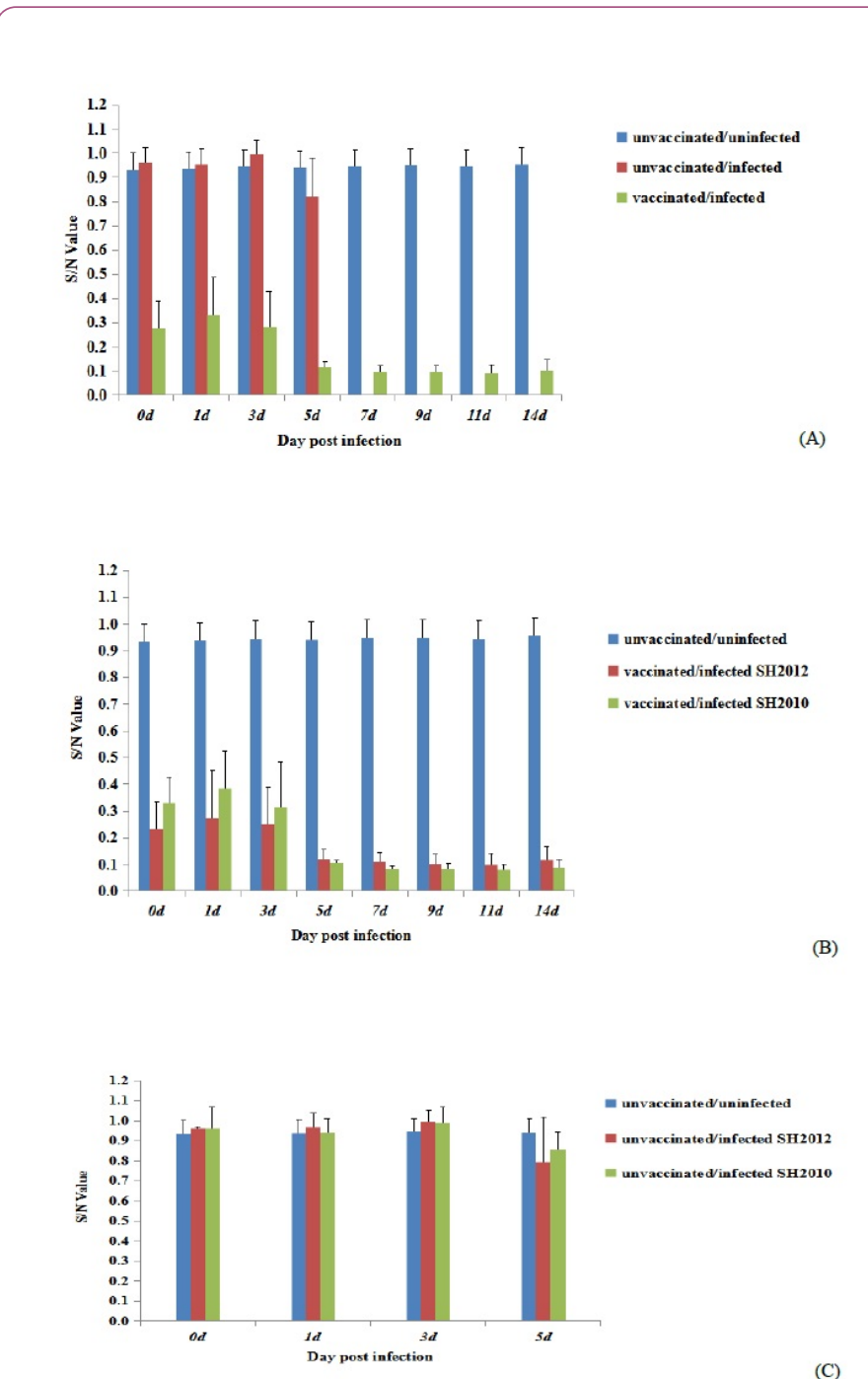

Figure 3: PRV gB-specific antibodies were detected with PRV gB Test Kit (IDEXX, USA) based on a type of competitive inhibition ELISA. If $\mathrm{S} / \mathrm{N} \leq$ cutoff value, the sample is positive for gB antibodies. $S / N=$ sample to negative control ratio. Cutoff value $=0.60$. Data in $(A),(B)$, and $(C)$ were presented as mean \pm SD. 


\section{Detection of viral DNA and virus isolation}

Nasal swab samples (143) were collected from the sheep of all three groups. All nasal swab samples remained negative based on virus isolation and polymerase chain reaction (PCR) analysis.

Seven samples of the brain and seven of the internal organs were each collected from groups $A$ and $B$. The highest levels of viral DNA in the three unvaccinated/infected sheep in group $A$ were detected in the brain (cycle threshold (ct) 16.32) and internal organs (ct 23.73). Viral titers in brain samples peaked at 6 DPI with $10^{3.0}$ tissue culture infective doses $\left(\mathrm{TCID}_{50}\right)$ per $\mathrm{ml}$. In contrast, the three unvaccinat-ed/infected sheep in group $B$ yielded the highest levels of viral DNA at 5 DPI in the brain (ct 13.43) and internal organs (ct 20.56). Viral titers in brain samples peaked at 5 DPI with $10^{3.25}$ TCID50 per ml.

\section{Gross pathology and histopathological findings}

At postmortem and on histopathological examination of the sheep, multiple lesions were observed on several organs (Figures 4A-4F). Pathologic examination showed that the most striking gross lesions were consolidated in the lungs, with extensive redness caused by congestion and hemorrhage (Figure 4B), in addition to slight cerebral edema (Figure 4A). In addition, several areas of hemorrhage were observed in the kidneys (Figure 4C).

On histopathological examination, severe hemorrhage and congestion were observed in the lungs (Figure 4E), as well as neuronal degeneration and necrosis in the brain (Figure 4D), and hemorrhage in the renal medulla (Figure 4F).

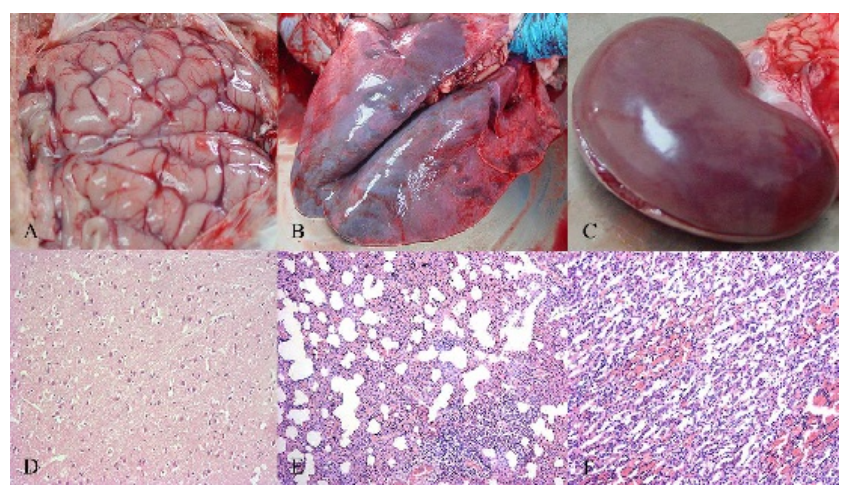

Figure 4: Severe damage to multiple organs in experimental, infected sheep by postmortem and histopathological examinations. (A) Brain, showing slight encephalic edema. (B) lung, showing extensive redness arising from congestion and hemorrhage. (C) Kidney, showing many bleeding spots. (D) Brain, neuronal degeneration and necrosis (E) lung, pulmonary hemorrhage and congestion. (F) Kidney, Hemorrhage in renal medulla. H\&E stain, $\times 200$.

\section{Discussion}

Vaccination of pigs with attenuated live or inactivated vaccines is widely performed to minimize po-tentially huge economic losses due to PRV infection [16-18]. For many years, pseudorabies has been efficiently controlled by vaccination in China. However, a sudden outbreak on many PRV-vaccinated pig farms in China occurred towards the end of 2011. This suggested that the PR vaccine was not effective against wild-type PR infection. According to recent studies, the causative agent of the outbreak was a PRV variant that showed enhanced pathogenicity $[3,7,8,10]$. In the present study, the PRV strain SH2012 was isolated in 2012 from an infected pig farm in Shanghai, China that used the typical vaccine. The vaccinated pigs were not completely protected against infection and exhibited obvious clinical signs of disease, similar to the typical signs observed in the field, suggesting that the virulence of the newly isolated PRV strains differed from that of the original strains. This virulence might have caused the death of infected pigs.

The $\mathrm{gC}$ gene of PRV is known to vary between strains, and is the most efficacious target for vi-rus-neutralizing antibodies [19]. In the present study, the two PRV isolates and other strains previously isolated in China, were observed to have both insertions of seven amino acids and many amino acid mutations in the $\mathrm{gC}$ gene. Among these, the E43A mutation also occurred in the Bartha, Becker, and Kaplan strains [20]. Furthermore, the $\mathrm{gC}$ gene is most widely used for phylogenetic analysis of PRV strains mainly because the sequence is prone to variation $[5,11,21]$. Recent reports have revealed that PRV can be classified into two major genotypes: genotype I (European and American strains) and gen-otype II (Chinese strains) [15]. The two isolates in the present study could be classified in genotype II. SH2012 was closely and independently clustered together with the emergent strains isolated after 2011. However, the earlier strain Fa (isolated in 1964) and SH2010 comprised a small subgroup and showed divergence from the emergent strains, including SH2012.

In the present study, two strains of PRV (SH2010 and SH2012) were used to infect sheep. Based on the clinical signs, variations in body temperature, and the highest levels of viral DNA in the unvac-cinated/infected sheep, the emerging PRV variant strain SH2012 is more highly virulent than the tradi-tional PRV SH2010 strain. The results demonstrated that the Bartha-K61 vaccine could effectively protect against lethal challenge with the traditional PRV SH2010 strain, but not against the emerging PRV variant strain $\mathrm{SH} 2012$. These findings are consistent with those of other studies $[3,11]$.

The data generated by our study will increase our understanding of the differences in pathogenicity between the traditional PRV SH2010 strain and the emerging PRV variant strain SH2012. The patho-genicity of the emerging PRV variant strain should be studied in greater depth and compared to that of the traditional PRV strains. Understanding the molecular mechanisms driving PRV virulence in China is important, and further experimental studies will be required in the near future.

\section{Acknowledgments}

This publication was supported by grants from the Shanghai Scientific Agricultural Tacking Program (no. 2014 7-3-3) and the Shanghai Agricultural Young Talents Program (no. 2014 2-9). 


\section{References}

1. Muller T, Hahn EC, Tottewitz F, Kramer M, Klupp BG, et al. (2011) Pseu-dorabies virus in wild swine: a global perspective. Arch Virol 156: 1691-1705.

2. Yuan QZ, Wen Y, Li YX, Li ZR, Nan X (1983) The pseudorabies vaccination research.I: Pseudorabies attenuated vaccine research. Chin J Prev Veterinary Med 1: 1-6 (in Chinese).

3. An TQ, Peng JM, Tian ZJ, Zhao HY, Li N, Liu YM, et al. (2013) Pseudorabies virus variant in Bartha-K61-vaccinated pigs, China, 2012. Emerg Infect Dis 19: 1749-1755.

4. Tong GZ, Chen HC (1999) Pseudorabies epidemic status and control measures in China. Chin J Vet Sci 19: 1-2 (in Chinese).

5. Gu Z, Hou C, Sun H, Yang W, Dong J, et al. (2015) Emergence of highly virulent pseudora-bies virus in southern China. The Canadian Journal of Veterinary Research 79: 221-228.

6. Wang Y, Qiao S, Li X, Xie W, Guo J, et al. (2015) Molecular epidemiology of outbreak-associated pseudorabies virus (PRV) strains in central China. Virus Genes 50: 401-409.

7. Wu R, Bai C, Sun J, Chang S, Zhang X (2013) Emergence of virulent pseudorabies virus infection in Northern China. J Vet Sci 14: 363-365.

8. Yu X, Zhou Z, Hu D, Zhang Q, Han T, Li X, Gu X, Yuan L, Zhang S, Wang B, Qu P, Liu J, Zhai X, Tian K (2014) Pathogenic pseudorabies virus, China, 2012. Emerg Infect Dis 20:102-104.

9. Tong W, Zhang QZ, Zheng H, Liu F, Jiang YF, et al. (2013) Identification and characterization of a Pseudorabies virus isolated from a dead piglet born to vaccinated sow. Chin J Animal Infect Dis 21: $1 \mathrm{e} 7$.

10. Luo Y, Li N, Cong X, Wang CH, Du M, et al. (2014) Pathogenicity and genomic characterization of a pseudorabies virus variant isolated from Bartha-K61-vaccinated swine population in China. Vet Microbiol 174: 107-115.

11. Wang CH, Yuan J, Qin HY, Luo Y, Cong X, et al. (2014) A novel gEdeleted pseudorabies virus (PRV) provides rapid and complete protection from lethal challenge with the PRV variant emerging in Bartha-K61-vaccinated swine population in China. Vaccine 32: 3379-3385.
12. Muller T, Klupp BG, Freuling C, Hoffman B, Mojcicz M, et al. (2010) Characterization of pseudorabies virus of wild boar originating from Europe. Epi-demiol Infect 138: 1590-1600.

13. Tamura K, Peterson D, Peterson N, Stecher G, Nei M, et al. (2011) MEGA5: Molecular evolu-tionary genetics analysis using maximum likelihood, evolutionary distance, and maximum parsimo-ny methods. Mol Biol Evol 28: 2731-2739.

14. Chinese Ministry of Agriculture (2015) Quality standards for veterinary biological products of Peo-ple's Republic of China; Beijing: China Agriculture Press, pp 93-94 (in Chinese).

15. Ye C, Zhang QZ, Tian ZJ, Zheng H, Zhao K, et al. (2015) Ge-nomic characterization of emergent pseudorabies virus in China reveals marked sequence diver-gence:Evidence for the existence of two major genotypes. Virology 483: 32-43.

16. Cunha EMS, Peixoto ZMP, Kotait I, Bersano JG (1998) Efficacy of an inactivated Aujeszky's dis-ease virus vaccine: experimental infection of pigs. Brazilian Journal of Veterinary Research and Animal Science 35: 188-194.

17. Mettenleiter TC, Klupp BG, Weiland F, Visser N (1994) Characterization of a quadruple glycopro-tein-deleted pseudorabies virus mutant for use as a biologically safe live virus vaccine. J Gen Virol 75: 1723-1733.

18. Rooij EM, Moonen-Leusen HW, de Visser YE, Middel WG, Boersma WJ, et al. (2006) A DNA vaccine coding for $g B$ and $g D$ of pseudorabies virus (suid herpes type 1) primes the immune system in the presence of maternal immunity more efficiently than conventional vaccines. Vaccine 24: 1264-1273.

19. Brittle EE, Reynolds AE, Enquist LW (2004) Two modes of pseudorabies virus neuroinvasion and lethality in mice. J Virol 78: 12951-12963.

20. Szpara ML, Tafuri YR, Parsons L, Shamim SR, Verstrepen KJ, Legendre M, Enquist LW (2011) A wide extent of inter-strain diversity in virulent and vaccine strains of alphaherpesviruses. PLoS Pathog 7: e1002282.

21. Goldberg TL, Weigel RM, Hahn EC, Scherba G (2011) Comparative utility of restriction fragment length polymorphism analysis and gene sequencing to the molecular epidemiological investigation of a viral outbreak. Epidemiol Infect 126: 415-424. 